\title{
BMJ Open Psychometrics properties of the Team Interaction Scale and influencing factors of team interaction of tertiary hospital physicians in China: a cross- sectional study
}

Wenwen Song, ${ }^{1}$ Honghe Li, ${ }^{1}$ Ning Ding, ${ }^{1}$ Weiyue Zhao, ${ }^{1}$ Lin Shi, ${ }^{2}$ Deliang Wen ${ }^{\oplus 1}$

To cite: Song W, Li H, Ding N, et al. Psychometrics properties of the Team Interaction Scale and influencing factors of team interaction of tertiary hospital physicians in China: a crosssectional study. BMJ Open 2019;9:e026162. doi:10.1136/ bmjopen-2018-026162

- Prepublication history and additional material for this paper are available online. To view these files, please visit the journal online (http://dx.doi. org/10.1136/bmjopen-2018026162).

Received 24 August 2018 Revised 5 March 2019 Accepted 6 June 2019
Check for updates

(C) Author(s) (or their employer(s)) 2019. Re-use permitted under CC BY-NC. No commercial re-use. See rights and permissions. Published by BMJ.

${ }^{1}$ Institute for International Health Professions Education and Research, China Medical University, Shenyang, Liaoning, China

${ }^{2}$ Medical Insurance Department, Shenyang Tenth People's Hospital, Shenyang, Liaoning, China

Correspondence to Professor Deliang Wen; dlwen@cmu.edu.cn

\section{ABSTRACT}

Objectives To administer a cross-cultural adaptation of the Team Interaction Scale (TIS), test its psychometric properties and investigate influencing factors of team interactions in a physician population in Chinese tertiary hospitals.

Design Cross-sectional survey.

Settings Two rounds of surveys, a pilot and a large sampling survey, were conducted in two and nine tertiary hospitals, respectively, in Liaoning Province, China.

Participants In the pilot survey, 363 of 390 physicians sampled were included in the analysis, resulting in an effective response rate of $93.08 \%$. In the large sampling survey, the effective response rate was $89.10 \%$ (3653 of 4100 physicians)

Outcome measures The TIS and a short version of a burn-out scale were administrated to assess the physician's team interaction and burn-out. Psychometric properties of TIS were tested by confirmatory factor analysis (CFA), exploratory factor analysis (EFA) and internal consistency analysis. Gender, age, discipline, education level, professional title, hospital scale and burnout were explored as influencing factors with independent sample $t$-tests, one-way analyses of variance and a correlation analysis.

Results Based on CFA, a 17-item modified scale was developed following the pilot survey. In the large sampling survey, EFA was conducted with half of the samples, producing six dimensions: 'Communication', 'Coordination', 'Mutual help', 'Team goals', 'Work norms' and 'Cohesion and conflict resolution'. Fit of the modified model was confirmed by CFA with the other half of the samples (root mean square error of approximation $=0.067$, Comparative Fit Index $=0.98$, Normed Fit Index=0.97, Goodness of Fit Index=0.94, Adjusted Goodness of Fit Index=0.92). A high Cronbach's $\alpha$ coefficient of 0.98 demonstrated reliability of the modified scale. The Team Interaction Score was significantly lower in younger physicians, in men, in paediatricians and in physicians from larger-scale tertiary hospitals. Team Interaction Scores were negatively associated with burn-out.

Conclusions The adapted TIS, containing 17 items and six dimensions, was reliable and valid for Chinese tertiary hospital physicians. To address physician burn-out, team interaction should be highlighted.
Strengths and limitations of this study

- To the best of our knowledge, this is the first study with a sufficient and representative sample in China to introduce a comprehensive dimensional structure to assess the interaction of the physician's team.

- This study extends the research on physicians' team interaction by identifying the potential influential factors and provides empirical research evidence for team interaction improvement.

- The design of this two-survey study ensured the reliability and validity of the results.

- The evaluation of the team interaction was self-report, which may be subject to reporting bias.

- This study was cross-sectional, so the causal relationships between team interaction and the influencing factors were not clear.

\section{BACKGROUND}

Teamwork has been confirmed to be fundamental to team efficiency, physician wellbeing and patient safety, and is generally acknowledged as the core of patient-centred medical reforms. ${ }^{1-5}$ Team interaction is a dynamic, changing sequence of social actions between individuals that includes such activities as monitoring, coordination and communication, and is a dominant process of teamwork. ${ }^{6}$ Furthermore, the dynamics of team interaction are associated with team efficiency and output in healthcare teams, ${ }^{7}$ and as such, we should pay more attention to healthcare team interactions.

Good team interactions are fundamental for physician teams around the world. Modern healthcare demands successful physician teamwork, particularly with interprofessional and cross-setting teamwork, ${ }^{8}$ which are becoming increasingly demanding of the coordination and communication processes in the healthcare teams. Accordingly, 
physician team interaction is a dominant factor of high-efficiency healthcare.

Healthy team interaction is particularly important for Chinese physician teams. Chinese tertiary hospitals admit a majority of relatively serious cases on the spectrum of disease, resulting in a high-intensity work environment, contributing to a documented high rate of physician burn-out in Chinese tertiary hospitals. ${ }^{9}$ Since teamwork has been reported to be a factor protecting from physician burn-out, Chinese tertiary hospital physicians could benefit from better team interaction, enabling release of emotional exhaustion and improved team efficiency. ${ }^{3} 10$

To date, however, there is lack of empirical research on team interactions. ${ }^{611}$ Although emphasis has been placed on the assessment of team interaction and an accepted scale has been designed for the assessment of team interactions (eg, the Team Performance Scale), ${ }^{6}{ }^{12}$ the conceptual framework of team interaction in the healthcare field has not been well explored. The Lechler Scale of assessing team interaction measures the perceived social interaction among innovative and entrepreneurial team members. This scale, based on the theoretical concept of Hoegl, was widely used for entrepreneurial team research. ${ }^{13}{ }^{14}$ While there have been studies on teamwork in China, ${ }^{15}$ there are no known existing studies that examine healthcare team interactions. Indeed, only a few instruments are particularly oriented towards the measurement of team interaction within clinical physicians in China and abroad and none have proposed a comprehensive core dimension of team interaction in the field of healthcare. ${ }^{616}$

The aim of this study, therefore, is to introduce an instrument, to confirm its ability to effectively measure the physician's perceived team interaction, to investigate the current status of physician team interaction and to explore potential influencing factors. Although the sound dimensional structure of assessing social interaction proposed by Lechler has been widespread in innovative team research, this is the first known time that it has been adapted and validated for a healthcare team. ${ }^{14}$ We made it as the scale of assessing team interactions for physician populations and called it the Team Interaction Scale (TIS). The psychometric properties of the scale were verified in the Chinese tertiary hospital physician population through two rounds of surveys. As burn-out is negatively associated with teamwork, ${ }^{17}$ the relationship between burn-out and team interaction was also explored in this study.

The introduction of this scale allows us to possess a valid tool for assessing the perceived team interaction of physicians in the healthcare team, to understand the present status of the team interaction and to gain knowledge on the influencing factors of the team interaction, thus assisting healthcare policymakers and administrators to promote healthcare quality as well as physician wellbeing from a long-term, team perspective.

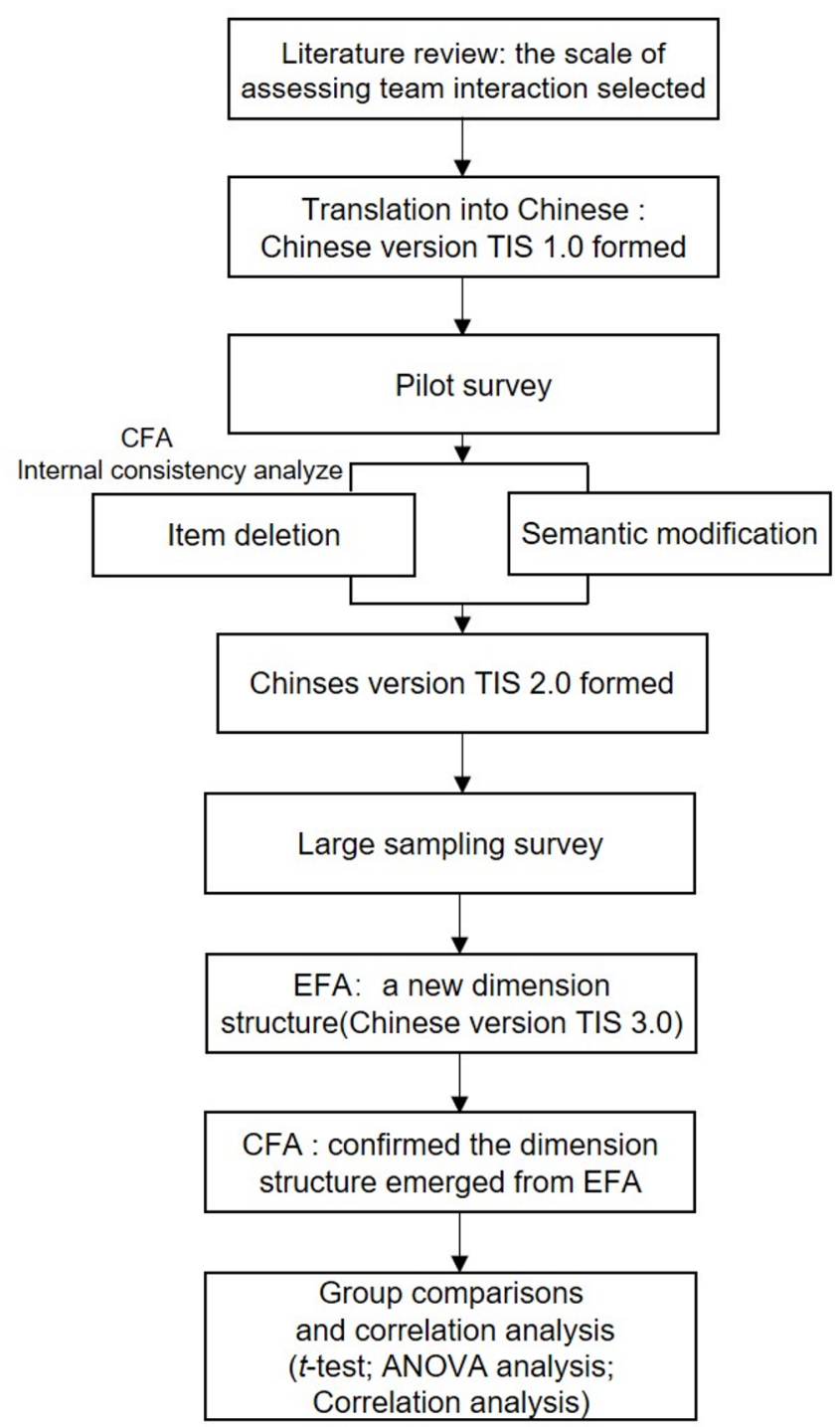

Figure 1 Steps and methods of cross-cultural adaptation and validation of Team Interaction Scale (TIS). ANOVA, analysis of variance; CFA, comparative fit analysis; EFA, exploratory fit analysis.

\section{METHODS}

Two rounds of surveys were conducted for cross-cultural adaptation and validation of this study (see figure 1 for steps and methods).

\section{Study design}

Questionnaire of the pilot survey

TIS was translated independently into Chinese by one graduate student (WS) and one faculty member (ND) from the China Medical University and was subsequently compared and reviewed by five experienced clinical experts to confirm the cultural and academic relevance, which yielded the initial translated version. This version was then back-translated by two faculty members (WZ and HL) from the China Medical University, both of whom were blind to the initial English version scale. The comparison between the back-translated scale and the original English scale led to minor revisions, 
resulting in Chinese V.1.0 of TIS. This version of the scale contained six factors: 'Communication', 'Coordination', 'Mutual support', 'Work norms (effort)', 'Cohesion' and 'Conflict resolution'. All 31 self-report items were positively worded with a seven-point Likert-type scale, scored from 1 (strongly disagree) to 7 (strongly agree). The total score was calculated from the sum of all items, ranging from 31 to 217, with a higher score indicating better team interaction.

A sociodemographic questionnaire was also designed and applied to acquire personal characteristics of the physicians, including gender, age, discipline and education level.

\section{Study sample of the pilot survey}

In December 2016, 390 physicians from two tertiary hospitals in Liaoning Province, China, were invited to participate in the pilot survey. In order to provide a representative sample of clinical physicians in these tertiary hospitals, a randomised cluster sampling method was applied. Physicians from several disciplines, including internal medicine, surgery, obstetrics and gynaecology, paediatrics and other disciplines, including pathology, anaesthesiology, ear-nose-throat (ENT), stomatology, ophthalmology, radiology, ultrasound, intensive care unit (ICU) and traditional Chinese medicine departments were randomly chosen. The total number of physicians in each discipline was used as the sampling weight.

\section{Questionnaire of the large sampling survey}

We maintained the seven-point Likert-type scale for the modified scale and also included a sociodemographic questionnaire including gender, age, discipline and education level. A total score was calculated, ranging from 17 to 119 , with a higher score indicating better team interaction. To gather information for psychometric properties of the scale and to confirm the relationship between team interaction and physician burn-out, a two-item burn-out scale, previously confirmed to be reliable and valid, was also applied in the large sampling survey. ${ }^{18}$

\section{Study sample of the large sampling survey}

The large sampling survey was conducted including 4100 physicians from nine tertiary hospitals in Liaoning in February 2017. Considering the potential heterogeneity in the hospitals with different qualities, a stratified cluster sampling method was used to select a representative sample from all physicians in Liaoning. Nine out of 37 tertiary hospitals in Liaoning Province were randomly chosen, including three of the top 20 tertiary hospitals in North-East China (larger scale of tertiary hospitals) and 6 ordinary tertiary hospitals. In each tertiary hospital, physicians from internal medicine, surgery, obstetrics and gynaecology, paediatrics, and other disciplines, including the departments of pathology, anaesthesiology, ENT, stomatology, ophthalmology, radiology, ultrasound, the ICU, and the traditional Chinese medicine departments were randomly chosen. The total number of physicians in each discipline was also used as the sampling weight.

\section{Procedure and ethics statement}

The participants were selected and voluntarily participated in the study. We were permitted to distribute the paper questionnaire off-line and maintain contact with each clinical physician team to ensure the survey was completed. Participants were able to consult trained researchers with any questions regarding the survey. Each participant was assured of confidentiality and signed a written informed consent prior to completing the questionnaire. The coded self-report questionnaires were completed independently in approximately $10 \mathrm{~min}$. The participants were not compensated and were able to withdraw from the survey at any time.

Both surveys in this study were approved with the understanding that all information would be used only for our study and would be kept confidential.

\section{Statistical analysis}

The data from the pilot survey were analysed using confirmatory factor analysis (CFA) to test the psychometric properties and to subsequently modify Lechler's model. Then the data from the large sampling survey were equally and randomly divided into two parts. Exploratory factor analysis (EFA) and CFA were performed with one of the two parts, separately. In other words, CFA was used to confirm the factor structure that emerged from EFA in a distinct data set.

Specifically, to evaluate the model fit in CFA, we used maximum likelihood estimation and referred to various fit indices, including the $\chi^{2}$ value, root mean square error of approximation (RMSEA), Goodness of Fit Index (GFI), Adjusted Goodness of Fit Index (AGFI), Comparative Fit Index (CFI) and Normed Fit Index (NFI). If GFI, AGFI, CFI and NFI were greater than 0.90 and RMSEA was less than 0.08 , the fit of the model was deemed acceptable. ${ }^{19}$ If the model poorly fit the data, the item causing high Modification Index (MI) values would be revised or even deleted. For example, if all the authors agreed the items suffered from cultural gaps, the entry would be deleted. After deletion of any item, the model was rerun to calculate new fit indices and an updated MI. The process was iterated until an acceptable model fit was achieved.

Before conducting EFA, a Kaiser-Meyer-Olkin (KMO) analysis was performed to test the adoption of the factor analysis. In EFA, principal component factor extraction and varimax rotation were employed to illustrate the underlying dimensional structure of the Chinese version of TIS and the maximum likelihood method was used to estimate the model. Eigenvalues, relative magnitude and direction of factor loadings were all examined to explain variance and communality.

Cronbach's $\alpha$ coefficients were calculated to estimate the internal consistency of each dimension and of the overall scale. An $\alpha$ coefficient of higher than 0.70 was 
considered acceptable and coefficients higher than 0.90 represent an extremely high level of reliability.

The correlations between the overall score of perceived team interaction and gender, age, discipline, education level, professional title and hospital scale were evaluated with t-tests and a univariate analysis of variance with all the samples in the large sampling survey. Effect sizes, including Cohen's $\mathrm{d}$ and partial $\eta$ squared $\left(\eta_{p}^{2}\right)$, were also reported to illustrate the practical meaning of the difference. The effect sizes were referred to as small with $\mathrm{d}=0.20$ and $\eta_{p}{ }^{2}=0.01$, as medium with $\mathrm{d}=0.50$ and $\eta_{p}{ }^{2}=0.06$, and as large with $\mathrm{d}=0.80$ and $\eta_{p}{ }^{2}=0.14 .{ }^{20}$ Pearson's correlation coefficients were calculated to explore the potential correlation between burn-out and perceived team interaction on each dimension.

Missing values were imputed with the medians of the corresponding entries. All the data analyses were implemented via SPSS V.23 and AMOS V.24, and $p<0.05$ was considered statistically significant.

\section{Patient and public involvement}

Neither patients nor the public were involved in the design or conduct of the study.

\section{RESULTS}

\section{Preliminary psychometrics of the scale}

Pilot survey

In the pilot survey, 390 questionnaires were distributed, with 363 completed questionnaires returned for an effective response rate of $93.08 \%$. Men accounted for $51.50 \%$ of the sample and $50.70 \%$ of the participants were between the ages of 31 years and 40 years (table 1 ).

The results of the CFA in the pilot survey indicated a poor fit, with $\chi^{2}$ for the original 31-item scale of 2090.43, GFI $=0.71$, AGFI $=0.66$, NFI $=0.87, \mathrm{CFI}=0.89$ and RMSEA $=0.11$, suggesting that the original model didn't perform well in the Chinese physician population. We made semantic modifications and deleted some items per the MI and feedback from physicians and experts, resulting in Chinese V.2.0 of the TIS with 17 items. The revised scale yielded a $\chi^{2}$ value of 327.13 with acceptable fit indices (GFI $=0.91$, AGFI $=0.86, \mathrm{NFI}=0.96, \mathrm{CFI}=0.97$, RMSEA=0.08). The factor loadings before and after modification are shown in an online additional file 1 .

In the pilot survey, internal consistency for each dimension and the overall scale were tested with the 17 -item model after the modification process. All $\alpha$ coefficients were higher than 0.80 , ranging from 0.90 to 0.98 , indicating that all the items provided adequate contributions to the scale after the modification.

\section{Large sampling survey}

The 17-item TIS was distributed to physicians within nine representative hospitals in Liaoning Province, China. Among the 4100 questionnaires distributed, 3653 pieces were completed, leading to an effective response rate of

\begin{tabular}{llc}
\hline $\begin{array}{l}\text { Table 1 The distribution of demographic variables for the } \\
\text { pilot survey }\end{array}$ & N (\%) \\
\hline Demographics & Category & $187(51.50)$ \\
\hline Gender & Male & $176(48.50)$ \\
& Female & $84(23.10)$ \\
Age, years & $\leq 30$ & $184(50.70)$ \\
& $31-40$ & $70(19.30)$ \\
& $41-50$ & $24(6.60)$ \\
Discipline & $51-60$ & $1(0.30)$ \\
& $61-70$ & $139(38.30)$ \\
& Internal medicine & $150(41.30)$ \\
& Surgery & $24(6.60)$ \\
& Obstetrics and & $11(3.00)$ \\
& Gynaecology & $39(10.70)$ \\
& Paediatrics & $172(47.40)$ \\
& Others & $179(49.30)$ \\
Education level & Doctors & $11(3.00)$ \\
& Masters & $1(0.30)$ \\
& Bachelors & $98(27.2)$ \\
& Others & $138(38.3)$ \\
Professional title & Primary title & $93(25.8)$ \\
& Intermediate title & $31(8.6)$ \\
\hline & Associate professor & \\
& Professor &
\end{tabular}

Other disciplines include the departments of pathology, anaesthesiology, ear-nose-throat (ENT), stomatology, ophthalmology, radiology, ultrasound, the intensive care unit (ICU), and the traditional Chinese medicine department.

Other education level includes a college degree.

$89.10 \%$. The distribution of all demographic variables in the two parts of the sample were similar (see table 2).

A KMO analysis was performed among half of the samples, yielding an index of 0.98 . The result of Bartlett test of sphericity was significant at $36101.81 \quad(\mathrm{p}<0.01)$. Therefore, we conducted EFA using a principal component factor extraction with varimax rotation to explore the potential factor model (see table 3 for results). Six factors emerged, called 'Communication', 'Coordination', 'Mutual help', 'Team goals', 'Work norms' and 'Cohesion and conflict resolution' (Chinese V.3.0, see online additional file 2 for details). The overall 17 -item model accounted for $87.20 \%$ of the variance (see table 3 ).

The 17-item model that emerged from EFA was verified with CFA with another half of the samples, yielding an excellent model fit with $\chi^{2}=955.75$, RMSEA $=0.067$, and CFI, NFI, GFI and AGFI scores all higher than 0.90, at $0.98,0.97,0.94,0.92$, respectively. The factor loadings were all higher than 0.80 (details in online additional file 1), suggesting that all the items provided adequate contributions to each factor. The path diagram of the confirmed model is presented in figure 2. 


\begin{tabular}{|c|c|c|c|}
\hline $\begin{array}{l}\text { Demographic } \\
\text { variables }\end{array}$ & Category & $\begin{array}{l}\mathbf{N}(\%) \\
\text { (part 1) }\end{array}$ & $\begin{array}{l}\mathbf{N}(\%) \\
\text { (part 2) }\end{array}$ \\
\hline \multirow[t]{2}{*}{ Gender } & Male & $890(48.80)$ & $887(48.50)$ \\
\hline & Female & 935 (51.20) & $941(51.50)$ \\
\hline \multirow[t]{4}{*}{ Age, years } & $21-30$ & $313(17.20)$ & 363 (19.90) \\
\hline & $31-40$ & 837 (45.90) & 773 (42.30) \\
\hline & $41-50$ & $397(21.80)$ & 432 (23.60) \\
\hline & $\geq 50$ & $278(15.20)$ & $260(14.20)$ \\
\hline \multirow[t]{5}{*}{ Discipline } & $\begin{array}{l}\text { Internal } \\
\text { medicine }\end{array}$ & 767 (42.00) & 777 (42.50) \\
\hline & Surgery & $610(33.40)$ & 597 (32.70) \\
\hline & $\begin{array}{l}\text { Obstetrics and } \\
\text { gynaecology }\end{array}$ & $93(5.10)$ & $96(5.30)$ \\
\hline & Paediatrics & 57 (3.10) & $56(3.10)$ \\
\hline & Others & 298 (16.30) & 302 (16.50) \\
\hline \multirow{4}{*}{$\begin{array}{l}\text { Education } \\
\text { level }\end{array}$} & Doctors & $376(20.60)$ & $374(20.50)$ \\
\hline & Masters & $839(46.00)$ & $879(48.10)$ \\
\hline & Bachelors & $590(32.30)$ & $562(30.70)$ \\
\hline & Others & $20(1.10)$ & $13(0.70)$ \\
\hline \multirow{4}{*}{$\begin{array}{l}\text { Professional } \\
\text { title }\end{array}$} & Primary title & $528(28.9)$ & $520(28.4)$ \\
\hline & Intermediate title & $617(33.8)$ & $619(33.9)$ \\
\hline & $\begin{array}{l}\text { Associate } \\
\text { professor }\end{array}$ & $317(17.4)$ & 342 (18.7) \\
\hline & Professor & 363 (19.9) & $346(18.9)$ \\
\hline \multirow[t]{2}{*}{ Hospital scale } & $\begin{array}{l}\text { North-east top } \\
20 \text { hospitals }\end{array}$ & 879 (48.20) & $928(50.80)$ \\
\hline & $\begin{array}{l}\text { Ordinary tertiary } \\
\text { hospital }\end{array}$ & $946(51.80)$ & $900(49.20)$ \\
\hline
\end{tabular}

Other disciplines include the departments of pathology, anaesthesiology, ear-nose-throat (ENT), stomatology, ophthalmology, radiology, ultrasound, the intensive care unit (ICU), and the traditional Chinese medicine department. Other education level includes a college degree.

Further, as the significant correlations between team interaction factors were observed (see online additional file 3), a second-order CFA was performed to test the potential structure of the scale. The path diagram of the second-order factor structure is presented in figure 3 . The regression weights of the six first-order factors were all greater than 0.90 . The model fit of the second-order factor structure was acceptable with $\chi^{2}=1473.22$, RMSEA $=0.081$, $\mathrm{CFI}=0.96, \mathrm{NFI}=0.96$, GFI $=0.91$ and $\mathrm{AGFI}=0.88$.

All Cronbach's $\alpha$ coefficients of the six dimensions and the overall scale of the final 17 -item TIS were higher than 0.85 , ranging from 0.87 to 0.98 (see table 4 ).

\section{Influencing factors}

Group comparisons

The perceived Team Interaction Score demonstrated significant differences in gender, age, discipline and hospital scale, but no significant difference between different education levels and professional title (see table 5 for results).

Female physicians perceived better team interaction than male physicians $(t=-3.85, \mathrm{p}<0.05)$ and there was a generally positive trend with respect to age and perception of team dynamics, specifically, physicians between 21 years and 30 years of age perceived the lowest team interaction and physicians older than 40 years rated a distinctly better team interaction $(F=5.33, \mathrm{p}<0.01)$. Additionally, the perceived Team Interaction Score was significantly higher in those practising internal medicine than in surgeons, while paediatricians scored the lowest of all professions $(F=6.73, \mathrm{p}<0.01)$. Furthermore, team interactions were rated better in ordinary tertiary hospitals than in the north-east top 20 tertiary hospitals (hospitals of a larger scale) $(t=-2.93, \mathrm{p}<0.01)$.

Effect sizes showed that Cohen's $d$ of gender and hospital scale were 0.21 and 0.10 , indicating a non-overlap of $14.7 \%$ and $7.7 \%$, respectively, in the two distributions. Using $\eta_{p}{ }^{2}$ as the measure of association, the values 0.004 , $0.007,0.001,0.002$ showed a relatively small difference of perceived team interaction among different age groups and disciplines.

\section{Correlation analysis}

The overall Team Interaction Score was inversely related to burn-out and the six factors 'Communication', 'Coordination', 'Mutual help', 'Team goals', 'Work norms' and 'Cohesion and conflict resolution' were all significantly associated with burn-out (see table 6).

\section{DISCUSSION}

The aim of this study is to cross-culturally adapt and validate TIS among physicians in Chinese tertiary hospitals and to explore potential influencing factors of team interactions. The structure of the scale was adjusted based on the results of the CFA in the pilot survey and EFA in the large sampling survey, with the new model verified through CFA in the large sampling survey. The results suggest that the Chinese version of the scale consisting of six dimensions and 17 items was reliable. The perceived Team Interaction Score was significantly lower in male physicians, paediatricians and physicians from larger-scale tertiary hospitals. Additionally, physicians perceived significantly better team interaction associated with longevity on the team and physician burn-out was negatively related to perception of team interaction.

In the pilot survey, the model fit indices of the original structure did not meet the criterion for moderate construct validity, suggesting that the cross-cultural validity of the original instrument was low and was inappropriate to apply to physicians in the Chinese tertiary hospital. ${ }^{21}{ }^{22}$ Team interaction is determined mostly by interpersonal factors, which may be influenced by politics, the economy and culture context. ${ }^{6}$ As this scale of assessing team interaction was first introduced into a 
Table 3 Exploratory factor analysis for the 17-item Team Interaction Scale (TIS)

Rotated factor coefficients

'Cohesion

Items *

and conflict 'Work 'Mutual 'Team

\begin{tabular}{lllllll} 
Items * & resolution' & 'Coordination' & norms' & help' & 'Communication' & goals' \\
\hline $\begin{array}{l}\text { 16. The team members solve conflicts and } \\
\text { disagreements within the team completely }\end{array}$ & $\mathbf{0 . 7 7}$ & 0.32 & 0.28 & 0.23 & 0.21 & 0.21 \\
$\begin{array}{l}\text { 17.Disagreements between the team members } \\
\text { are solved rapidly }\end{array}$ & $\mathbf{0 . 7 2}$ & 0.31 & 0.33 & 0.18 & 0.23 & 0.26 \\
$\begin{array}{l}\text { 15. Strong cohesion is a characteristic of the } \\
\text { team }\end{array}$ & $\mathbf{0 . 6 7}$ & 0.29 & 0.40 & 0.30 & 0.18 & 0.19 \\
$\begin{array}{l}\text { 14. Working in the team has the highest priority } \\
\text { f. }\end{array}$ & $\mathbf{0 . 5 9}$ & 0.22 & 0.48 & 0.32 & 0.25 & 0.18
\end{tabular}

for every team member (in comparison with

other jobs and private life)

\begin{tabular}{|c|c|c|c|c|c|c|}
\hline $\begin{array}{l}\text { 4. The team members adjust closely the } \\
\text { processing of their tasks }\end{array}$ & 0.35 & 0.70 & 0.27 & 0.28 & 0.21 & 0.26 \\
\hline $\begin{array}{l}\text { 3. The team members share opinions and } \\
\text { information spontaneously }\end{array}$ & 0.32 & 0.69 & 0.31 & 0.22 & 0.34 & 0.17 \\
\hline $\begin{array}{l}\text { 5. Within the team related tasks are well } \\
\text { coordinated. }\end{array}$ & 0.35 & 0.61 & 0.23 & 0.35 & 0.25 & 0.37 \\
\hline $\begin{array}{l}\text { 11. The team members share the workload of } \\
\text { the team equally }\end{array}$ & 0.32 & 0.28 & 0.71 & 0.13 & 0.22 & 0.36 \\
\hline $\begin{array}{l}\text { 12. Every team member works as best as she/ } \\
\text { he can in order to achieve the team's goals }\end{array}$ & 0.38 & 0.36 & 0.70 & 0.25 & 0.14 & 0.18 \\
\hline $\begin{array}{l}\text { 13. Every team member is completely } \\
\text { integrated in the team }\end{array}$ & 0.38 & 0.21 & 0.68 & 0.36 & 0.26 & 0.15 \\
\hline $\begin{array}{l}\text { 7. Discussions among the team members are } \\
\text { always constructive and beneficial }\end{array}$ & 0.33 & 0.35 & 0.30 & 0.66 & 0.28 & 0.23 \\
\hline $\begin{array}{l}\text { 6. The team members support and } \\
\text { complement each other as well as they can }\end{array}$ & 0.31 & 0.51 & 0.31 & 0.54 & 0.19 & 0.24 \\
\hline $\begin{array}{l}\text { 8. Proposals and contributions of the team } \\
\text { members are always respected }\end{array}$ & 0.33 & 0.38 & 0.29 & 0.53 & 0.23 & 0.42 \\
\hline $\begin{array}{l}\text { 1. The team members communicate intensively } \\
\text { with each other }\end{array}$ & 0.30 & 0.32 & 0.22 & 0.24 & 0.75 & 0.27 \\
\hline $\begin{array}{l}\text { 2. I'm completely content with the exactness of } \\
\text { information provided by other team members }\end{array}$ & 0.23 & 0.55 & 0.38 & 0.23 & 0.56 & 0.11 \\
\hline $\begin{array}{l}\text { 9. The team members reach consensus in } \\
\text { every important issue }\end{array}$ & 0.30 & 0.29 & 0.31 & 0.39 & 0.25 & 0.63 \\
\hline $\begin{array}{l}\text { 10. Every team member perceives herself/ } \\
\text { himself as responsible for the clinical team's } \\
\text { goals }\end{array}$ & 0.43 & 0.34 & 0.41 & 0.20 & 0.25 & 0.54 \\
\hline$\%$ Variance & 19.87 & 17.89 & 17.60 & 12.02 & 10.13 & 9.70 \\
\hline
\end{tabular}

Coefficients in bold were higher than 0.50 .

*Items listed in accordance with the value of coefficients.

physician population and applied to a Chinese healthcare context, the target population difference and the cultural gap may have contributed to the dimensional structure being unadaptable, leading to the low validity of the scale. The dimensional structure of the modified 17-item scale was different from that of Lechler's original six-dimensional structure, with the addition of the 'Team goals' dimension and the combination of the 'Cohesion' and 'Conflict resolution' dimensions, but it retained the 'Communication', 'Coordination' and 'Work norms (effort)' dimensions. ${ }^{23-25}$ The two items in the newly formed dimension 'Team goals' contained wording for 'reaching consensus' and 'perception of responsibility for the team's goals', both highlighting the common goals in the team. Due to the content as well as the emphasis of team goals in team process research, the dimension was therefore named as 'Team goals'. ${ }^{26}$ The results implied that 'Team goals' was one of the core attributes of team 


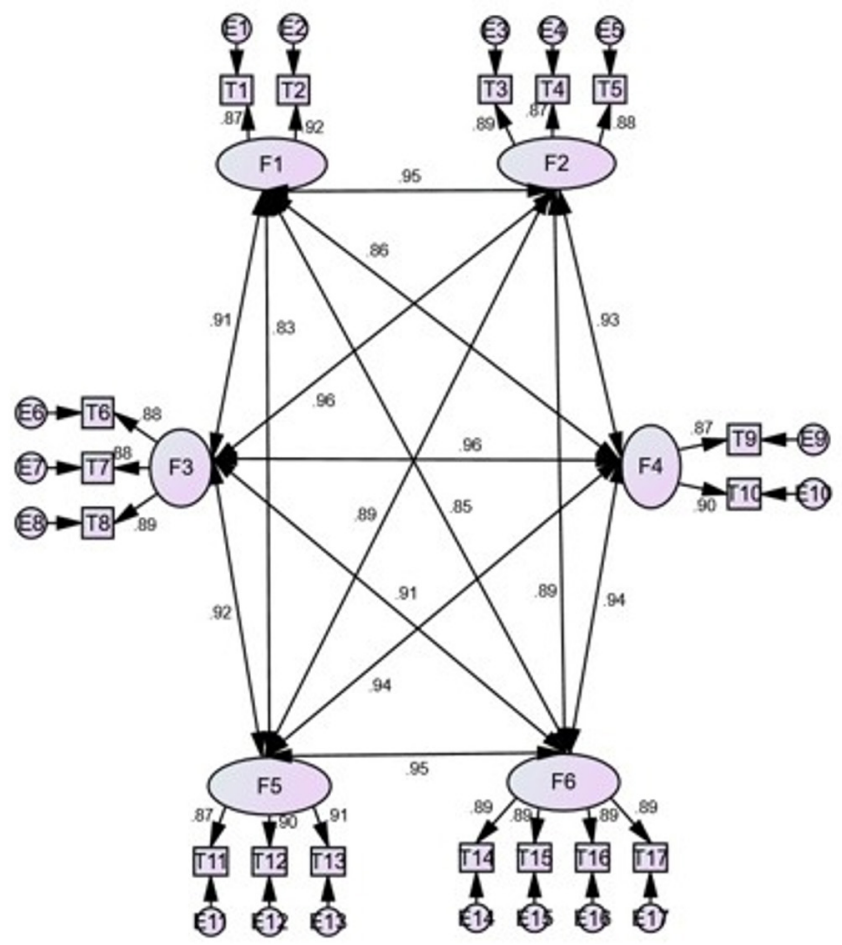

Figure 2 The path diagram of the 17-item Team Interaction Scale (TIS) model emerged from exploratory factor analysis (EFA).

interaction in Chinese tertiary hospital physician teams. Changes in the dimensional structure revealed that the connotation and manifestation of team interaction may be differentiated by the target population and culture context. Among the six dimensions of the modified scale, there are two dimensions consisting of two items each, which may have an influence on the reliability of the scale. However, the Cronbach's $\alpha$ coefficient of the 17 -item scale in the large sampling survey $(\alpha=0.98)$ was close to that in the pilot survey $(\alpha=0.98)$, which was an acceptable range for educational and psychological testing. ${ }^{25}$ Additionally, the $\alpha$ coefficients of each factor of the final scale were all higher than 0.80 , suggesting high internal consistency of TIS among Chinese tertiary hospital physicians. The high regression weights in second-order CFA supported that the second-order factor structure existed and the six subdimensions contributed equally to explain team interaction. The acceptable model fit indicated that team interaction could be manifested through the six subdimensions yielded by factorial analysis. In the future, we will apply the 17 -item short TIS as well as the 31 -item full scale at the same time, to compare the two instruments, validating the short version scale for use to examine physician team interaction.

The item mean score for the dimension 'Communication' was the lowest (mean=6.02, $\mathrm{SD}=1.04$ ), suggesting that physicians generally perceived poor communication within their teams. However, previous research has demonstrated that communication is a key component in the team process. ${ }^{27}$ Therefore, the administrators should

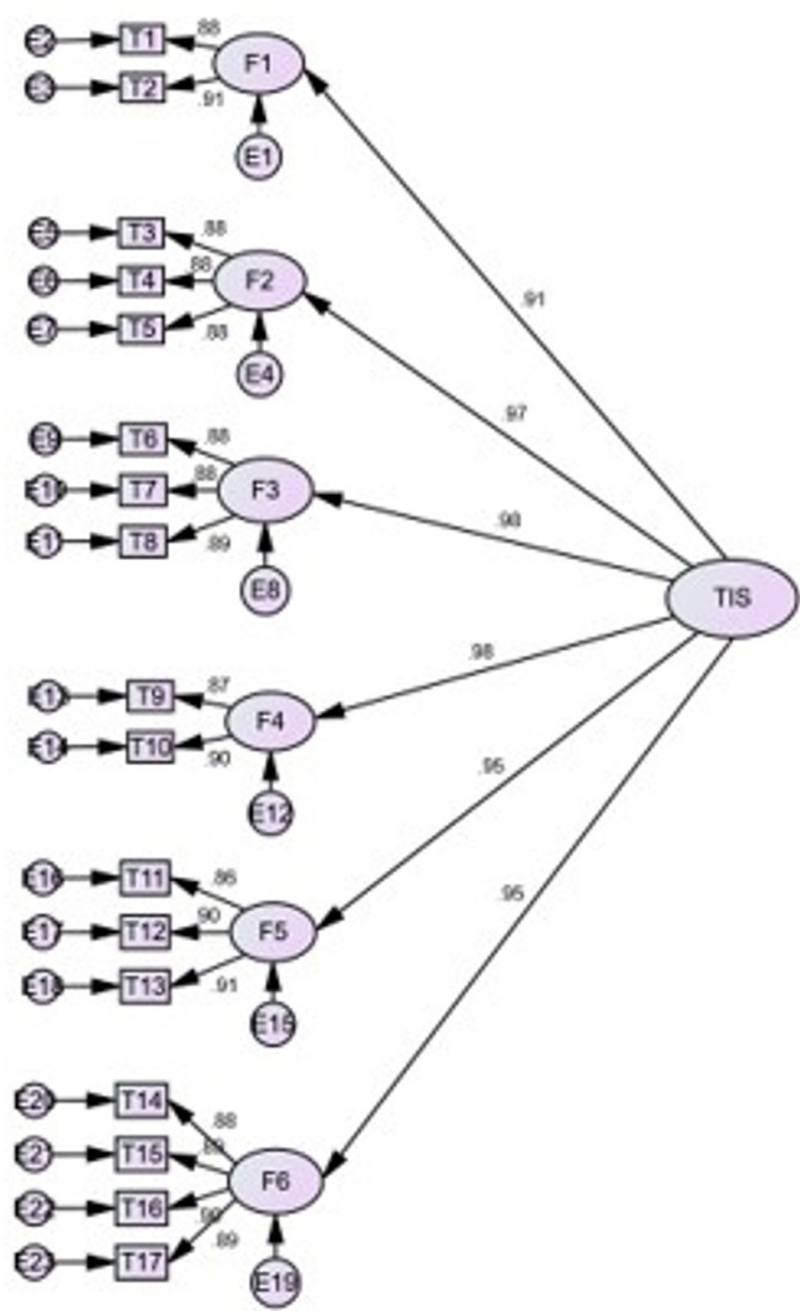

Figure 3 The path diagram of the second-order factor structure. TIS, Team Interaction Scale .

make a concerted effort to improve communication within teams, resulting in better team interaction.

Similar to other studies in which the female members tended to experience better team interaction, communication and team cohesion, the current study demonstrated that perceived team interaction was higher in women than in men. ${ }^{28}$ Women tend to be more relational, which may contribute to better team interaction. ${ }^{28}$ Physicians older than 40 years perceived significantly better team interaction, while those in their 20s perceived poorer interaction, suggesting that age is a positive predicting factor of perceived team interaction. Similar findings also illustrated that physicians and other health professionals appreciated better teamwork as working years increased. ${ }^{29-31}$ It may be that elder physicians tend to have more longevity within their teams, allowing them to be better integrated with the team compared with the younger physicians. Moreover, elder physicians are qualified in clinical skills, teamwork ability and other essential competencies, so they may be more respected and others may cooperate with them more, resulting in feelings of a better team interaction climate. ${ }^{30}$ According to the results 
Table 4 Cronbach's $\alpha$ coefficients and mean scores of the final 17 -item Team Interaction Scale (TIS) model

$\alpha$ coefficients of final model

(item number of each

\begin{tabular}{llcl} 
17-item TIS & domain) & Dimension mean (SD) & Item mean (SD) \\
\hline Communication & $0.88(2)$ & $12.05(2.07)$ & $6.02(1.04)$ \\
Coordination & $0.92(3)$ & $18.37(2.93)$ & $6.12(0.98)$ \\
Mutual help & $0.92(3)$ & $18.52(2.85)$ & $6.17(0.95)$ \\
Team goals & $0.87(2)$ & $12.36(1.94)$ & $6.18(0.97)$ \\
Work norms & $0.92(3)$ & $18.38(3.04)$ & $6.13(1.01)$ \\
$\begin{array}{l}\text { Cohesion and conflict } \\
\text { resolution }\end{array}$ & $0.94(4)$ & $24.63(3.92)$ & $6.16(0.98)$ \\
Overall & & & $6.13(0.91)$ \\
\end{tabular}

of our study, the administration should provide physicians younger than 40 years with more human care and growth opportunities.

In the current study, the Team Interaction Score was significantly different within disciplines, with paediatricians scoring the lowest. Facing the special patient groups of extreme age, paediatric physicians may encounter more challenges in teamwork. ${ }^{32}{ }^{33}$ First of all, the difficulty in coordination and cooperation with children brings obstacles to the physician's work, potentially increasing medical errors, hindering the team interaction within paediatric physicians. ${ }^{33-35}$ Additionally, a feature of the paediatrics discipline is that it often relies on multidisciplinary teamwork, which is more demanding of the physicians' teamwork competencies, thereby increasing potential issues with team interactions. ${ }^{33}$ Above all, particular attention should be paid to the team interaction of paediatric physicians. The physicians in ordinary tertiary hospitals rated a significantly better team interaction than the tertiary hospitals

Table 5 Group comparisons of the Team Interaction Score within demographic and working variables

\begin{tabular}{|c|c|c|c|c|c|}
\hline Variables & Category & Mean (SD) & $F / t$ & $P$ values & Effect size \\
\hline \multirow[t]{2}{*}{ Gender } & Male & $103.29(16.28)$ & $t=-3.85$ & $p<0.05$ & Cohen's $d=0.21$ \\
\hline & Female & 105.30 (14.72) & & & \\
\hline \multirow{2}{*}{ Age, years } & $31-40$ & $104.26(15.26)$ & & & \\
\hline & $41-50$ & $105.40(14.97)$ & & & \\
\hline \multirow{3}{*}{ Discipline } & Surgery & $102.64(16.81)$ & & & \\
\hline & $\begin{array}{l}\text { Obstetrics and } \\
\text { gynaecology }\end{array}$ & $105.55(12.81)$ & & & \\
\hline & Paediatrics & 101.75 (20.43) & & & \\
\hline \multirow{2}{*}{ Education level } & Bachelors & $104.87(17.57)$ & & & \\
\hline & Others & 105.44 (18.96) & & & \\
\hline \multirow[t]{4}{*}{ Professional title } & Primary title & $103.89(16.25)$ & $F=2.44$ & $p=0.062$ & $\eta_{p}^{2} \mathrm{P} 20.002$ \\
\hline & Intermediate title & $103.71(15.30)$ & & & \\
\hline & Associate professor & $104.82(15.13)$ & & & \\
\hline & Professor & $105.47(15.14)$ & & & \\
\hline \multirow[t]{2}{*}{ Hospital scale } & $\begin{array}{l}\text { North-east top } 20 \\
\text { hospitals }\end{array}$ & $103.55(14.00)$ & $t=-2.93$ & $p<0.01$ & Cohen's $d=0.10$ \\
\hline & $\begin{array}{l}\text { Ordinary tertiary } \\
\text { hospitals }\end{array}$ & $105.05(16.86)$ & & & \\
\hline
\end{tabular}

$\eta_{p}^{2}$, partial $\eta$ squared. 


\begin{tabular}{|c|c|c|c|c|c|c|c|}
\hline Variables & Communication & Coordination & Mutual help & Team goals & Work norms & resolution & Total score \\
\hline Burn-out & $-0.21^{*}$ & $-0.22^{*}$ & $-0.22^{*}$ & $-0.23^{*}$ & $-0.24^{*}$ & $-0.24^{*}$ & $-0.25^{\star}$ \\
\hline
\end{tabular}

*Denotes values significant at $\mathrm{p}<0.01$.

of a larger scale. In China, the larger scale of tertiary hospitals are faced with the most serious diseases on the disease spectrum, which demands better teamwork. Additionally, the physicians have higher demands on the team process, possibly contributing to the relatively lower Team Interaction Score. Another potential factor influencing team interactions is that the better tertiary hospitals usually have a larger group of physician teams, introducing more challenges to the interaction of the physicians within their teams. Furthermore, the physicians in the better tertiary hospitals are faced with a busier working environment, more critical cases and more medical errors, which may negatively influence the team process and exacerbate physician burn-out, risking the teamwork process. ${ }^{36}{ }^{37}$ Therefore, we suggest that more attention on team building needs to be paid in larger-scale tertiary hospitals.

Consistent with other reports that teamwork quality was related to health professional burn-out, the results of the correlation analysis in the current study suggest that physician burn-out was negatively associated with team interaction. We propose that improvement in the environment of team interaction may potentially relieve physician burn-out. ${ }^{3} 103839$ Therefore, physician wellbeing could be improved from the perspective of individual characteristics, and from working environments like team interaction. ${ }^{40}$

\section{Limitations}

This survey was implemented in only one province of China, which may impair the generalisation of our conclusions. However, the sample in this study was representative of this province and the demographic characteristics of the sample are quite similar with that of the national physician population shown $i^{41}$ the China Health and Family Planning Yearbook $2017 ;^{41}$ thus the limitation in representativeness may be negligible. In the final structure of TIS, there were two subscales only formed by two items, which may drive the instabilities of the scale in other samples. However, the validity of the scale has been fully demonstrated in the discussion section, and TIS has been confirmed a valid instrument for the assessment of team interaction. Furthermore, the causality of relationships between team interaction and influencing factors could not be determined due to the cross-sectional nature of the survey. Future studies could address this problem by tracking their participants.

\section{CONCLUSIONS}

In a population of Chinese tertiary hospital physicians, the adapted version of TIS containing 17 items and six dimensions is valid and reliable, taking into account the culture gap. The adapted version of TIS has the potential to be a valid tool for evaluating physicians' team interaction in other countries with similar cultures or similar healthcare contexts. Hospital administrators should pay increased attention to the environment of team interaction, which may help alleviate physician burn-out.

Acknowledgements The authors thank the developer, Thomas Lechler, for providing access to the scale of assessing team interaction and for his research assistance. The authors also thank all the hospitals and healthcare professionals, who participated in the survey, for their advice and assistance to the study. The authors also thank the translators of the questionnaire for their contribution to the translation process.

Contributors DW, WS and HL were responsible for the study design. WS, ND, HL and WZ were responsible for the translation of the questionnaire. WS, WZ and LS performed the data collection. WS, HL and ND contributed to the analysis and interpretation of the data and were involved in drafting the manuscript and revising it critically for important intellectual content, and gave final approval of the version to be published. All authors have read and approved the final manuscript.

Funding This study was funded by the Humanities and Social Sciences Research and Planning Fund Project of the Chinese Ministry of Education in 2014 (grant number:14YJAZH085). The funding has contributed to allocating time for use in the collection, analysis and interpretation of data, and in the writing of this manuscript.

Competing interests None declared.

Patient consent for publication Not required.

Ethics approval Bioethics Advisory Commission of China Medical University, Shenyang, China.

Provenance and peer review Not commissioned; externally peer reviewed.

Data sharing statement № additional data are available.

Open access This is an open access article distributed in accordance with the Creative Commons Attribution Non Commercial (CC BY-NC 4.0) license, which permits others to distribute, remix, adapt, build upon this work non-commercially, and license their derivative works on different terms, provided the original work is properly cited, appropriate credit is given, any changes made indicated, and the use is non-commercial. See: http://creativecommons.org/licenses/by-nc/4.0/.

\section{REFERENCES}

1. Pham JC, Aswani MS, Rosen M, et al. Reducing medical errors and adverse events. Annu Rev Med 2012;63:447-63.

2. Silva MC, Peduzzi M, Sangaleti CT, et al. Cross-cultural adaptation and validation of the teamwork climate scale. Rev Saude Publica 2016;50:50.

3. Estrynbehar M B, Heijden VD, Guetarni K, et al. Relevant indicators of psychosocial risks for the prevention of burnout in the hospital. Archives Des Maladies Professionnelles Et De Lenvironnement 2010:71.

4. Ouwens M, Hulscher M, Akkermans R, et al. The Team Climate Inventory: application in hospital teams and methodological considerations. Qual Saf Health Care 2008;17:275-80. 
5. Wahr JA, Prager RL, Abernathy JH, et al. Patient safety in the cardiac operating room: human factors and teamwork: a scientific statement from the American Heart Association. Circulation 2013;128:1139-69.

6. Healey AN, Undre S, Vincent CA. Developing observational measures of performance in surgical teams. Qual Saf Health Care 2004;13 Suppl 1:i33-i40.

7. Borges NJ, Thompson BM, Roman BJ, et al. Team interactions, and gender in medical students during a psychiatry clerkship. Academic psychiatry: the journal of the American Association of Directors of Psychiatric Residency Training and the Association for Academic Psychiatry 2015;39:661-3.

8. Mitchell P, Wynia M. Core principles \& values of effective team-based health care. Booksgooglecom 2012;1:79-80.

9. Wen J, Cheng Y, Hu X, et al. Workload, burnout, and medical mistakes among physicians in China: A cross-sectional study. Biosci Trends 2016;10:27-33

10. Vilà Falgueras M, Cruzate Muñoz C, Orfila Pernas F, et al. [Burnout and teamwork in primary care teams]. Aten Primaria 2015;47.

11. Delphin E, Davidson M. Teaching and evaluating group competency in systems-based practice in anesthesiology. Anesth Analg 2008;106:1837-43.

12. Marlow S, Bisbey T, Lacerenza C, et al. Performance measures for health care teams: A review. Small Group Res 2018;49:306-56.

13. Hoegl M, Gemuenden HG. Teamwork quality and the success of innovative projects: A theoretical concept and empirical evidence. Organization Science 2001;12:435-49.

14. Lechler T. Social interaction: A determinant of entrepreneurial team venture success. Small Business Economics 2001;16:263-78.

15. Cooper S, Cant R, Connell C, et al. Measuring teamwork performance: Validity testing of the Team Emergency Assessment Measure (TEAM) with clinical resuscitation teams. Resuscitation 2016;101:97-

16. Valentine MA, Nembhard IM, Edmondson AC. Measuring teamwork in health care settings: a review of survey instruments. Med Care 2015;53:16-30.

17. Rama-Maceiras P, Kranke P. Working conditions and professional wellbeing: a link easy to imagine but difficult to prove. Eur $J$ Anaesthesiol 2013;30:213-5.

18. West CP, Dyrbye LN, Sloan JA, et al. Single item measures of emotional exhaustion and depersonalization are useful for assessing burnout in medical professionals. $J$ Gen Intern Med 2009;24:1318-21.

19. Lt H, Bentler PM. Cutoff criteria for fit indexes in covariance structure anaysis: Conventional criteria versus new alternatives. Structural Equation Modeling 1999;6:1-55.

20. Lakens D. Calculating and reporting effect sizes to facilitate cumulative science: a practical primer for t-tests and ANOVAs. Front Psychol 2013;4:863.

21. Kroman SL, Roos EM, Bennell KL, et al. Measurement properties of performance-based outcome measures to assess physical function in young and middle-aged people known to be at high risk of hip and/or knee osteoarthritis: a systematic review. Osteoarthritis Cartilage 2014;22:26-39.

22. Terwee CB, Bot SD, de Boer MR, et al. Quality criteria were proposed for measurement properties of health status questionnaires. $J$ Clin Epidemiol 2007;60:34-42.
23. Dietz AS, Pronovost PJ, Mendez-Tellez PA, et al. A systematic review of teamwork in the intensive care unit: what do we know about teamwork, team tasks, and improvement strategies? J Crit Care 2014:29:908-14.

24. Kiesewetter J, Fischer MR, Jan Kiesewetter MRF. The teamwork assessment scale: A novel instrument to assess quality of undergraduate medical students' teamwork using the example of simulation-based ward-rounds. GMS Z Med Ausbild 2015;32.

25. Hull L, Bicknell C, Patel K, et al. Content validation and evaluation of an endovascular teamwork assessment tool. Eur J Vasc Endovasc Surg 2016;52:11-20.

26. Brennan SE, Bosch $\mathrm{M}$, Buchan $\mathrm{H}$, et al. Measuring team factors thought to influence the success of quality improvement in primary care: a systematic review of instruments. Implement Sci 2013;8:20.

27. Thornton BC, McCoy ED, Glover TW, et al. Interaction on health care teams. 1980. J Interprof Care 2007;21 Suppl 1(sup1):76-85.

28. Post $\mathrm{C}$. When is female leadership an advantage? Coordination requirements, team cohesion, and team interaction norms. J Organ Behav 2015;36:1153-75.

29. Shaw KN, Ruddy RM, Olsen CS, et al. Pediatric patient safety in emergency departments: unit characteristics and staff perceptions. Pediatrics 2009;124:485-93.

30. Raftopoulos V, Pavlakis A. Safety climate in 5 intensive care units: a nationwide hospital survey using the Greek-Cypriot version of the safety attitudes questionnaire. J Crit Care 2013;28:51-61.

31. Raftopoulos V, Savva N, Papadopoulou M. Safety culture in the maternity units: a census survey using the Safety Attitudes Questionnaire. BMC Health Serv Res 2011;11:238.

32. Kohn LT, Corrigan JM, Donaldson MS. To err is human: building a safer health system. Annales Francaises $D$ Anesthesie Et De Reanimation 2000;7:245-6.

33. Eppich WJ, Brannen M, Hunt EA. Team training: implications for emergency and critical care pediatrics. Curr Opin Pediatr 2008;20:255-60

34. Singh $\mathrm{H}$, Thomas EJ, Wilson $\mathrm{L}$, et al. Errors of diagnosis in pediatric practice: a multisite survey. Pediatrics 2010;126:70-

35. Swinney R, Yin L, Lee A, et al. The role of support staff in pediatric palliative care: their perceptions, training, and available resources. $J$ Palliat Care 2007;23:44-50.

36. Morey JC, Simon R, Jay GD, et al. Error reduction and performance improvement in the emergency department through formal teamwork training: evaluation results of the MedTeams project. Health Serv Res 2002;37:1553-81.

37. Hunziker S, Johansson AC, Tschan F, et al. Teamwork and leadership in cardiopulmonary resuscitation. J Am Coll Cardiol 2011;57:2381-8.

38. Welp A, Meier LL, Manser T. The interplay between teamwork, clinicians' emotional exhaustion, and clinician-rated patient safety: a longitudinal study. Crit Care 2016;20:110.

39. Profit J, Sharek PJ, Amspoker AB, et al. Burnout in the NICU setting and its relation to safety culture. BMJ Qual Saf 2014;23:806-13.

40. Dyrbye L, Shanafelt T. A narrative review on burnout experienced by medical students and residents. Med Educ 2016;50:132-49.

41. China Health and Family Planning Yearbook, 2017. 